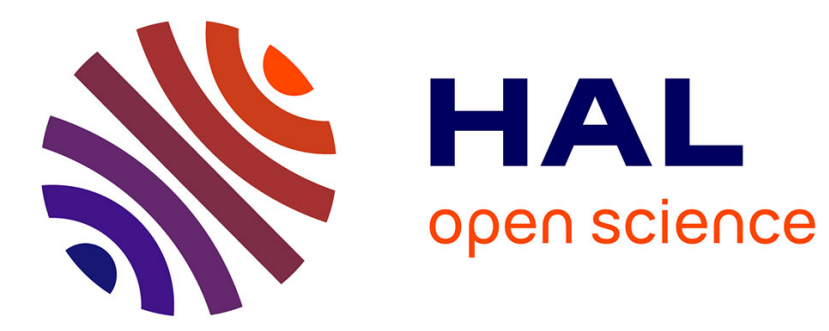

\title{
Homopaternités et gestation pour autrui
}

Martine Gross, Dominique Mehl

\section{To cite this version:}

Martine Gross, Dominique Mehl. Homopaternités et gestation pour autrui. Enfances Familles Générations, 2011, 14, pp.95-112. hal-00994138

\section{HAL Id: hal-00994138 \\ https://hal.science/hal-00994138}

Submitted on 21 May 2014

HAL is a multi-disciplinary open access archive for the deposit and dissemination of scientific research documents, whether they are published or not. The documents may come from teaching and research institutions in France or abroad, or from public or private research centers.
L'archive ouverte pluridisciplinaire HAL, est destinée au dépôt et à la diffusion de documents scientifiques de niveau recherche, publiés ou non, émanant des établissements d'enseignement et de recherche français ou étrangers, des laboratoires publics ou privés. 


\title{
Homopaternités et gestation pour autrui
}

\section{Martine Gross}

Sociologue, ingénieure de recherche en sciences sociales (CNRS), Centre d'études interdisciplinaires des faits religieux

gross@ehess.fr

\section{Dominique Mehl}

Sociologue, directrice de recherche (CNRS), Laboratoire communication et politique, Centre d'étude des mouvements sociaux

mehl@ehess.fr

\section{Résumé}

Le présent article se propose d'étudier les parcours de pères gays qui ont eu recours à une mère porteuse pour devenir parents. Nous explorons d'abord les étapes menant de l'acceptation de l'homosexualité à la réalisation du désir d'enfant en interrogeant la dimension conjugale ou individuelle du projet, les motivations présidant au choix $d u$ recours à une gestation pour autrui. Nous nous penchons ensuite sur les représentations de la maternité à l'oeuvre dans les discours et les relations qui s'instaurent entre les pères et ces «tiers de naissance » sans lesquels ces hommes ne seraient jamais devenus pères.

Mots clés: Homoparentalité, paternité, mère porteuse, donneuse d'ovocyte, désir d'enfant, gestation pour autrui, famille multicomposée

\begin{abstract}
This article follows the trajectories of gay fathers who chose to use a surrogate mother in order to become parents. First, we explore the steps leading from the acceptance of homosexuality to the fulfillment of the desire to father a child, examining the spousal or single aspect and the motivations behind the choice to use a surrogate. We then consider the social representations of motherhood evidenced in the discourse and the relationships that develop between the fathers and these 'birth others', without whom these men could not have become fathers.
\end{abstract}

Keywords: Gay parenting, paternity, surrogate mother, egg donor 


\section{Introduction}

Le terme « homoparentalité $»^{1}$ qualifie des situations familiales diverses : celles où les enfants sont arrivés alors que l'homosexualité de leurs parents était déjà vécue et assumée, et celles où ils sont nés d'une union hétérosexuelle avant que l'un des parents ne recompose un foyer avec une personne du même sexe. L'existence de parents homosexuels ne date pas de l'invention de l'homoparentalité, mais ils vivaient leur homosexualité en marge d'une union hétérosexuelle dont étaient issus leurs enfants. Les situations où la vie homosexuelle ne fait pas obstacle à l'expression d'un désir d'enfant ne se sont multipliées que depuis l'évolution du regard social porté sur l'homosexualité, notamment avec la reconnaissance légale du couple de même sexe.

En France, selon les données associatives ${ }^{2}$, la plupart des lesbiennes ont recours à l'insémination artificielle avec donneur (IAD), accessible et légale dans des pays voisins, tandis que les gays ont le plus souvent des enfants en coparentalité. Il s'agit pour un homme et une femme sans vie conjugale commune de concevoir et d'élever un enfant au sein de deux foyers : un foyer paternel et un foyer maternel. Dans la coparentalité, l'enfant a un père et une mère et peut avoir deux parents additionnels que sont le compagnon du père et la compagne de la mère. Un petit nombre d'hommes et de femmes se tournent vers l'adoption ${ }^{3}$ dans une démarche individuelle. Enfin, des hommes peuvent se rendre à l'étranger pour recourir à une gestation pour autrui (GPA). Au début des années 2000, très rares étaient les hommes qui optaient pour cette démarche. Ce n'est plus le cas aujourd'hui. Le nombre d'hommes qui se tournent vers la GPA ne cesse d'augmenter (Gross, 2009).

Si la crainte que l'homosexualité des parents puisse nuire à l'épanouissement de leurs enfants commence à se dissiper, les hommes et les femmes ne sont pas égaux face aux représentations sociales de la parentalité. Tout d'abord, devant la loi, la mère est celle qui donne naissance, tandis que le père ne peut se déclarer tel qu'en désignant la mère de son enfant. Être mère sans père se conçoit assez aisément, être père sans mère est beaucoup plus difficile. Ensuite, la compétence parentale est souvent synonyme de compétence maternelle. Si «materner», dans les représentations culturelles, rime avec féminité, "paterner», en revanche, est neutre de ce point de vue pour les hommes, voire dévirilisant, puisque ceux-ci peuvent même être considérés comme des "papas poules ». C'est surtout l'homoparentalité au masculin qui questionne la norme, avec au mieux une inquiétude liée à l'absence de mère et au pire une suspicion de pédophilie qui se profile chez les plus homophobes. Quand s'ajoute à l'homopaternité la question polémique du recours à la GPA, le débat peut devenir passionné entre ceux qui veulent un encadrement légal et éthique de la pratique et ceux qui prônent le maintien de l'interdit.

Excepté les thèses d'Emmanuel Gratton (Gratton, 2008) en sociologie clinique et de Flavio Tarnowski (Tarnowski, 2010) en anthropologie sociale, peu de travaux scientifiques francophones se sont penchés spécifiquement sur l'homopaternité. Le présent article se propose d'étudier les parcours de pères gays qui ont eu recours à une GPA pour devenir parent.

\footnotetext{
1 Le néologisme créé par l'Association des parents gays et lesbiens (APGL) en 1997 désigne les situations familiales dans lesquelles au moins un parent s'auto-identifie comme homosexuel.

2 http://www.apgl.fr/documents/enquete_apgl_2006-7.pdf

${ }^{3}$ En France, un enfant peut être adopté par une personne seule ou bien par un couple marié (donc hétérosexuel).
} 


\section{L'enquête}

L'étude a été menée de juillet 2009 à décembre 2009 dans la région parisienne et le Nord de la France à l'aide d'entretiens semi-directifs d'une heure trente à deux heures enregistrés et intégralement retranscrits. Les entretiens ont ensuite fait l'objet d'une analyse de contenu classique en sciences sociales. Chaque interview a été décryptée selon une grille thématique préétablie conforme à notre guide d'entretien : situation sociale de l'interviewé, situation conjugale, situation parentale, vécu de l'homosexualité, coming out, désir d'enfant, choix de la gestation pour autrui, représentation de la paternité, représentation de la maternité. Au fur et à mesure du dépouillement, cette grille a été affinée en fonction des réponses recueillies lors de l'enquête. Notamment, le rapport au lien biologique, l'engagement paternel et surtout le rapport à la maternité ont donné lieu à l'élaboration de typologies très fines et détaillées rendant compte de la diversité et de la richesse des discours des acteurs entendus lors de nos entretiens. En confrontant les réponses sur chaque item, nous avons dessiné une cartographie des représentations et relations d'expériences d'un point de vue exclusivement qualitatif. Aucune validation quantitative n'est intervenue dans cette construction compte tenu de la faiblesse numérique de l'échantillon et, également, du caractère tout à fait nouveau du phénomène qui induit, nous le verrons, une grande capacité d'innovation pour des pratiques qui ne sont pas encore stabilisées. Nous avons donc opté pour un traitement purement thématique respectant la diversité des points de vue et accordant à chaque expression le statut d'un témoignage emblématique. Nous avons rencontré 39 personnes, dont 34 en couples, 3 hommes en couple interviewés sans leur compagnon et 2 célibataires.

$\mathrm{Au}$ total, nous avons donc recueilli des témoignages concernant 22 familles, dont 13 couples avec enfants, 1 couple d'hommes chacun d'abord père de son côté avant de mener un projet parental commun encore en cours au moment de l'enquête, 2 célibataires avec enfants et 6 couples en projet.

Sur le plan sociodémographique, les enquêtés ont en moyenne 39 ans. Le plus jeune a 29 ans, le plus âgé a 65 ans. Ceux qui sont en projet sont plus jeunes, ils sont en moyenne âgés de 30 ans. Les couples sont en moyenne ensemble depuis 10 ans au moment de l'entretien, le couple le plus récent a 4 ans, le plus ancien, 20 ans. 5 hommes exercent une profession intermédiaire de l'enseignement ou de la santé, ou sont employés. Tous les autres sont cadres d'entreprise ou de la fonction publique, chefs d'entreprise ou exercent des professions libérales, intellectuelles et artistiques.

\section{Paternités}

\subsection{Entrer en paternité}

\subsubsection{Homosexualité et parenté}

Identifier son attirance sexuelle pour les garçons, pour les hommes. L'accepter déjà pour soi. Se sentir bizarre, différent. S'interroger sur la profondeur du phénomène et sur sa durabilité : inconstance passagère? Expérience initiatique qui appartiendra bientôt au passé, à sa jeunesse, mais ne persistera pas à l'âge adulte? Ou inclination fondamentale et irréversible? Nommer, reconnaître, accepter, puis assumer. Cette dernière étape est la plus difficile à franchir, car elle ne dépend pas que de soi. Elle se noue dans le rapport au monde extérieur. Or la société est loin d'être apaisée et la réprobation qui accompagne de tout temps l'homosexualité pèse sur chaque histoire singulière. Toujours la méfiance reste tapie. Ou tout simplement l'incompréhension, le sentiment d'étrangeté face à des personnes qui s'écartent du mode d'attraction sexuelle considéré comme naturel. L'homosexuel a été, est et demeure un être à part 
vis-à-vis de la société. Affronter le regard de celle-ci s'avère en conséquence extrêmement intimidant, voire périlleux. L'évolution des mœurs, les revendications égalitaires portées par les mouvements gays et lesbiens dans la foulée de la révolte soixante-huitarde ont contribué à atténuer les préjugés. Le débat sur le PACS (pacte civil de solidarité) et la visibilité acquise par les couples et familles homoparentales ont légitimé une façon d'être, de vivre et de partager qui demeurait enfouie dans le secret des vies privées et se donnait peu à voir au grand jour. Cette tolérance accrue, cependant, n'est encore que partielle comme en attestent nos interlocuteurs.

Les hommes que nous avons rencontrés ont pour la plupart vécu de façon complexe, chaotique et douloureuse leur découverte, puis l'acceptation et enfin la révélation de leur préférence sexuelle. Hommes de la quarantaine, ils sont venus au monde à l'heure où les mœurs se transforment, la parole sur le sexe se libère, mais la réprobation demeure très prégnante. Aussi ont-ils eu beaucoup de mal à s'assumer eux-mêmes, puis à se faire reconnaître autour d'eux.

Le récit d'Oscar, 37 ans, est véritablement emblématique du vécu de cette génération. Il se souvient :

Jusqu'au lycée, donc jusqu'à 18 ans, j'avais une attirance pour les garçons sans pouvoir mettre encore le nom. Jusqu'à 18 ans, j'étais amoureux d'une fille. C'était un amour platonique, il n'y aurait jamais eu de passage à l'acte, mais on s'entendait très, très bien et je n'arrêtais pas de penser à elle. Ensuite, à partir du BTS ${ }^{4}$, là les garçons, ça a été vraiment concret. Là, j'ai su que c'était ma vie, que je m'orientais vers les garçons et non pas vers les filles. Mais ça restait caché, même si je savais que les gens s'en doutaient. Ce n'était pas public. Il faut dire que c'était difficile. J'étais dans un milieu peut-être un peu macho. Et en plus, il faut se remettre dans le contexte. On était au tout, tout début des années 90 . Je pense que des jeunes de 18, 20 ans, maintenant, vivent peut-être mieux leur sexualité, enfin j'espère. Ça dépend peut-être des lieux aussi. En province, ça peut être encore dur.

Henri, la quarantaine aujourd'hui, déroule son histoire :

Je crois que j'ai toujours su que j'étais homo. Simplement, pendant l'adolescence, je pensais que c'était réversible! Qu'on pouvait vraiment choisir. Donc, j'ai vécu en étant un peu homo et un peu hétéro à la fois. Jusqu'à l'âge de 20, 22 ans. À partir de 22 ans, j'ai fait le choix de vivre pleinement mon homosexualité. Parce qu'à 22 ans, quand on a une relation de couple avec une jeune femme, dans son esprit c'est : le mariage, les enfants, tout s'enchaîne. Et là, pour moi, ça devient alors une certitude, je ne me voyais pas faire ma vie avec une femme. Et je me suis dit : puisque je suis sûr de ne pas être hétéro ou de ne pas vouloir une vie hétéro, alors là, c'est certain, je suis homo.

Comme eux, la quarantaine de gays qui se sont retrouvés avec nous pour évoquer leur paternité témoignent en général d'une prise de conscience peu précoce de leurs préférences en matière d'orientation sexuelle. D'une inclination difficile à assumer, d'autant que le tabou demeure extrêmement lourd. D'ailleurs, un bon tiers d'entre eux a d'abord, ou de façon concomitante, vécu des expériences amoureuses avec l'autre

${ }^{4}$ Brevet de technicien supérieur 
sexe, soit épisodiques, soit plus stables, avant de se «convertir » en toute certitude à une exclusivité masculine.

Une des premières conséquences du caractère houleux de leurs trajectoires de vie : des coming out relativement tardifs. Ils ne s'opèrent quasiment jamais en même temps que leur propre prise de conscience. Très rarement lors de leur vie adolescente, de collège, de lycée, de faculté. En réalité, pour la plupart, la parole sur ce sujet, notamment au sein de la famille, ne se déploiera qu'au moment où eux-mêmes choisissent de s'installer en couple. La révélation s'opère seulement lorsqu'un compagnon peut aussi être présenté. Quand le fils pense pouvoir attester devant ses parents d'une vie choisie et stable. À l'heure où la vie à deux prend tellement d'importance qu'elle ne peut plus être cachée sans amputer une dimension essentielle de leur identité. Il arrive même que leur coming out ne s'opère qu'avec l'engagement dans leur projet d'enfant. Comme si après avoir réfléchi, enquêté, puis engagé des démarches pour devenir pères, ils pouvaient enfin se présenter à leurs propres parents.

Henri a fait assez vite son coming out auprès de ses amis, mais pas de sa famille : « Ma famille, je voulais un peu la préserver. Mon idée était que je ferais mon coming out quand j'aurais une relation stable avec un homme. Pour leur dire : oui, je suis peut-être homo, mais je n'ai pas une vie délurée comme vous voyez à la télé, j'ai une vie stable avec quelqu'un et je suis très heureux comme ça. »

Ce lent cheminement, depuis la prise de conscience pour soi-même de son attirance pour les personnes de sexe masculin jusqu'à l'installation en couple. Du renoncement à une vie hétérosexuelle jusqu'à l'annonce aux entourages, notamment familiaux. De la certitude sur son identité à l'affichage public de celle-ci. De la peur du rejet à la décision d'affronter le regard d'autrui quand le couple prend forme ou même plus tard, quand l'idée de devenir père prend corps. De la hantise des réactions familiales à la surprise de rencontrer une certaine tolérance. Ce chemin-là est emprunté par la plupart de nos interlocuteurs.

Pourtant, d'autres scénarios ont aussi cours. Un père en solo d'un petit garçon n'a jamais fait son coming out. Tout le monde le pense hétérosexuel et séparé de la mère de son enfant. Il reconnait cependant que, s'il s'était installé avec un conjoint, il en aurait parlé. Pour quelques-uns d'entre eux, leur homosexualité assumée a eu pour conséquence la rupture avec leur famille. Et si les réactions des parents sont certes atténuées par l'annonce de l'enfant à venir, leur acceptation n'est cependant pas dénuée de déception.

Les histoires de ces hommes ayant opté pour la vie commune, puis pour un projet parental témoignent cependant d'une évolution sensible des représentations, des valeurs, des normes qui inspirent la société contemporaine. Car, et les plus jeunes d'entre eux en attestent le mieux, leur rapport à leur choix sexuel et leur désir d'entrer en parenté signent aussi la banalisation de l'homosexualité ou du moins son décollage de l'image originaire de l'insoumis.

Clément a seulement trente ans. Il affiche une vision de sa vie moins empreinte de souffrance, moins stigmatisée :

Je ne vis pas du tout caché. Au contraire, particulièrement ouvert. J'ai une photo de mon copain en fond d'écran de mon ordinateur. Je suis plutôt volontairement très out. Il faut dire que dans la pub, l'homosexualité n'est pas difficile à assumer. Le plus important, c'est que je veux être comme tout le monde. Je me sens complètement dans cette nouvelle génération du droit à l'indifférence plutôt que du droit à la différence. 
Et puis, avec humour, il suggère d'inverser l'image du rebelle.

On n'est pas un couple très libre, mais peut-être un peu plus que la moyenne. Peut-être qu'on a assimilé une forme de liberté qui va un peu, traditionnellement, historiquement, avec l'homosexualité. Nos amis homos ne sentent pas ce désir d'enfant du tout et ne sont pas du tout en couple, plutôt dans une consommation assez importante. Ils se moquent gentiment de nous quand on va à la campagne avec la belle famille. On devient presque aussi chiant que des hétéros. D'un certain côté, le fait de faire ce projet qui est un peu fou, ça ne me déplaît pas. Je me dis qu'aujourd'hui, c'est peut-être ce choix qui est un peu subversif.

\subsubsection{Désir d'enfant}

Le désir d'enfant des hommes a été jusqu'à présent peu étudié. Les pères gays que nous avons rencontrés expriment surtout un primat du désir de parentalité sur le désir d'engendrement. Une projection en tant que futur parent au quotidien de la vie de leurs enfants plutôt qu'un besoin intime de reproduction et de transmission. Ils fantasment plus volontiers sur les élans du cœur que sur ceux du corps.

Gérard formule clairement ce que tous expriment :

Moi, c'est plutôt une idée d'être parent, parce qu'en l'occurrence je ne suis pas le père biologique de notre fils et je pense que ça ne me perturbe pas vraiment. Donc, c'est plutôt l'idée d'être parent, plutôt que de me reproduire. Quand j'étais enfant, quand je me projetais dans l'avenir, j'aurais aimé avoir plein d'enfants. À l'époque, pour moi, avoir des enfants faisait partie des bonheurs de la vie. C'était quelque chose de profond.

Gérard, en affichant cette représentation prévalente de lui-même comme éducateur, accompagnateur, guide de sa progéniture, est tout à fait représentatif d'un sentiment partagé par les parents gay que nous avons rencontrés. Ceux-ci évoquent en toute priorité leur projection dans une parentalité à venir. Ils se sont toujours vus avec autour d'eux des enfants à élever, à guider dans la vie. Ils se propulsent dans un avenir où prime l'idée d'exercer un rôle parental au quotidien, avec la responsabilité domestique et éducative qui incombe aux gestionnaires d'un foyer. L'imaginaire de la paternité en acte supplante clairement le sentiment d'un destin corporel ou générationnel. Leur projet s'adosse d'abord au souhait de faire famille. Leur vœu s'ancre davantage dans une représentation de leur vie en société que dans l'aspiration à un accomplissement personnel. C'est moins le cheminement de l'enfantement que son aboutissement qui mobilise leur énergie, façonne leur personnalité.

Pierre, qui exprime un fort besoin d'engagement corporel, fait figure d'exception dans ce tableau lorsqu'il raconte que s'il l'avait pu, il aurait volontiers porté son enfant.

Et puis, quelques-uns, en sus de leur désir de paternage, expriment aussi des rêves qui font écho à ceux formulés par tout candidat à la parenté, homme ou femme, hétérosexuel ou homosexuel. Aspiration à panser des blessures familiales. Tel est le cas pour Jean-Louis qui espère fonder «la famille idéale » qu'il n'a pas eue, ayant grandi entre des parents désunis avec qui les liens se sont rompus après l'adolescence. Volonté de transmission. Comme Henri qui voudrait que sa vie ne se résume pas à son travail, qu'elle se prolonge à la génération suivante. Ou encore, inscription générationnelle et historique. Ainsi pour Frédéric chez qui cette thématique est primordiale. Juif dont une partie de la famille a été exterminée pendant la guerre, il 
projette dans l'enfantement son refus de l'anéantissement d'un peuple, sa volonté d'œuvrer pour « une renaissance après la mort ».

Quel que soit le chemin personnel qui finalement légitime leur désir d'enfant, la grande majorité d'entre eux reconnaissent toutefois avoir, un temps, fait le deuil de leur paternité. Leur expérience commune est, initialement, celle du renoncement. Dénégation, refoulement, choix de vie subi ou assumé, soumission obligée aux normes dominantes : toutes les variantes se conjuguent et s'additionnent pour aboutir à mettre en veilleuse ou oublier leur souhait de devenir père.

Pour tous ceux qui finalement vont revenir vers l'enfantement et désenfouir leur désir, le déclenchement a lieu avec l'entrée en conjugalité. Seuls quatre pères gay rencontrés au cours de cette enquête ont mené leur projet en solo. Tous les autres ne sont parvenus à reformuler pour eux-mêmes une hypothèse parentale qu'après la rencontre d'un compagnon. Leur installation commune, mais aussi leur projection dans l'avenir en tant que couple aspirant à la stabilité libèrent alors le refoulé.

Paul en témoigne :

À 26 ans, j'avais vraiment super envie d'avoir des gamins, mais je ne pouvais pas assouvir cette envie. Et puis, ça s'est tari, ça s'est estompé. Jusqu'à ce qu'on se rencontre. À partir du moment où on a été en couple, ça a forcément demandé du temps. Ce n'était pas quelque chose qu'on allait faire après un mois de vie commune. C'est quelque chose qui devait être construit, qui devait être le résultat d'une volonté commune.

Ainsi, paradoxalement, le débat sur le PACS et la loi qui l'instaure, entièrement conçue en vue de fermer la coparenté aux homosexuels tout en leur accordant des droits en tant que couple, a contribué à légitimer l'hypothèse homoparentale. Les polémiques qui accompagnent l'élaboration du dispositif législatif, agrémentées des multiples témoignages publics des parents de même sexe sur la vie familiale qu'ils ont néanmoins construite, ainsi que l'engagement des militants gays et lesbiens pour la cause homoparentale ont réactivé leur désir, leur ont conféré une réassurance et les ont confortés dans l'idée que leur abstinence paternelle pouvait n'avoir été qu'une parenthèse.

\subsection{Deux pères}

Lorsque la route vers l'enfantement est empruntée par un homme seul, tout concorde : la parenté biologique (il a donné son sperme), la parenté légale (il a reconnu l'enfant), la parenté éducative (il l'abrite et l'élève).

En revanche, pour ceux qui vivent en couple et comptent accueillir l'enfant ensemble, tout, au contraire, est décomposable. Chacun recherche une combinaison qui semble correspondre le mieux aux implications individuelles respectives d'un côté, à un projet commun de l'autre. Cependant pour tous, le point commun, le fil directeur de leur construction parentale réside dans l'exercice conjoint de la responsabilité éducative au quotidien. Que l'accomplissement de cette destinée paternelle soit porté davantage par l'un des membres du couple ou qu'il soit assumé par le duo, l'exigence reste la même : avoir la charge entière des « petits » dès leur arrivée au monde, vivre avec eux sans les partager avec d'autres partenaires de l'enfantement, les accompagner au long de leur maturation sans interférence d'autres adultes. La parentalité est, nous l'avons vu, au fondement de leur démarche. Elle guide l'ensemble des choix qu'ils sont amenés à faire. À l'inverse, ils savent qu'ils ne partageront pas la paternité légale, puisque 
l'homoparenté n'est pas reconnue en France. Ils auront donc à décider qui l'endosse. Quant à la paternité biologique, elle est également au choix.

Le scénario le plus répandu est celui où paternité biologique et paternité légale sont assumées par le même, la plupart du temps celui qui a été à l'origine du projet, celui qui y était le plus investi dès le départ, qui a parfois dû convaincre son compagnon, puis l'entraîner à ses côtés. Avec souvent l'idée que pour le deuxième enfant les rôles pourront s'inverser.

Cependant, même dans cette situation, il arrive que ce lien biologique ne soit pas très valorisé.

Clément en témoigne devant nous. Il sera le père biologique, car c'est lui qui a le plus envie de cet enfant, c'est lui qui s'en occupera le plus au quotidien, et parce qu'il est «moins accro au boulot» que son conjoint. Il sera aussi le père légal. S'il y avait un deuxième projet, ce serait possible que le père biologique soit son compagnon. Quoiqu'il ait le sentiment que ce serait plus simple que les enfants aient le même père biologique. À son avis, son compagnon est plus inquiet de n'avoir aucun lien légal avec l'enfant que de ne pas avoir de lien biologique :

Le lien biologique n'a pas une importance majeure. En mettant du mystère, on donne au lien biologique une importance trop forte par rapport à ce qu'il a en réalité. Au contraire, en étant dans une transparence la plus grande possible, on donne au lien biologique l'importance qu'il doit avoir, c'est-à-dire un rôle non primordial. Moi, je veux pouvoir retranscrire son histoire à cet enfant. Ça sera compliqué, mais au moins elle sera transparente. Qui sont tes parents biologiques? Cette femme, moi. Mais l'important, ce n'est pas le lien biologique, ce sont les parents qui t'ont voulu, qui t'aiment.

Quelques-uns, bien que moteurs du projet, laissent au conjoint le moins impliqué la fonction procréative. Marc ne souhaite pas être le père génétique, bien que ce soit chez lui que le désir d'enfant est le plus ancien et le plus affirmé. Il a des problèmes avec sa famille, s'entend très mal avec son frère également homosexuel et surtout, manque totalement d'estime de soi. «Je suis plein de névroses, je me déteste physiquement, je suis bourré de complexes. Alors que mon compagnon est beau, plein de confiance, hyperstable dans sa tête et a une fratrie, une famille très sympathique de quatre enfants. »

Marc se considère comme «le papa social», éducatif, mais ne veut pas apporter ses gènes.

Je me suis toujours dit que le sang, ça ne faisait rien, qu'il n'y avait pas besoin de sang pour que mon enfant m'aime autant que ses parents génétiques. Je n'ai jamais vraiment eu besoin de cette filiation-là. Donc, c'est ça en plus qui a convaincu mon ami de se lancer dans le projet : c'était mon choix de le laisser mettre la graine. Du coup, il a été plus motivé, il a mûri le projet, il l'a porté en lui.

Mais le départage ne traduit pas toujours des préoccupations existentielles. Il peut être aussi très prosaïque comme pour les couples qui ont désigné le père, car c'est lui qui maitrisait la langue et la culture du pays où se déroulerait la gestation.

Et puis, il arrive aussi que les deux hommes soient tous deux pères génétiques de deux jumeaux conçus chacun à partir d'embryons fécondés séparément par les deux hommes. Ils peuvent avoir vérifié, par un test ADN sur les enfants, les origines de ces 
derniers. Mais ils peuvent aussi ne pas vouloir savoir de qui (de l'un, de l'autre ou des deux?) ces jumeaux sont le fruit.

Ainsi, de même que lorsqu'ils évoquent leur désir d'enfant, lorsqu'ils choisissent celui qui va entrer dans le schéma reproductif, l'importance accordée au lien biologique, majeure pour quelques-uns, semble nettement plus ténue pour la plupart.

Le choix de recourir à une mère porteuse s'inscrit dans la logique de ces désirs et projections de paternité.

Tous ont songé à l'adoption, solution logique pour qui n'est pas foncièrement motivé par l'engagement procréatif, mais totalement investi dans un projet de parentalité. Pourtant, face à cette hypothèse, règne parmi eux le plus profond découragement. Bien que l'adoption soit ouverte aux célibataires, ils savent que l'agrément est rarement accordé aux hommes seuls. Et puis, comme les couples de femmes, ils répugnent à devoir dissimuler leur vie conjugale, à faire croire aux services sociaux qu'ils sont seuls, à supprimer le nom de l'autre sur la boîte aux lettres, à faire disparaitre de l'appartement toute trace du compagnon pour faire face aux exigences des enquêtes sociales préalables à l'autorisation d'adopter. Par ailleurs, ils éprouvent des craintes redoublées quant à la venue d'un enfant adoptable, car abandonné. Ils redoutent d'avoir à gérer une situation doublement complexe, celle d'une histoire enfantine déjà marquée par des blessures, à laquelle se surimposerait la difficulté à rassurer l'enfant inséré dans une combinaison parentale hors norme. En conséquence, parmi les personnes que nous avons rencontrées, presque toutes ont songé à l'adoption avant d'y renoncer. L'un s'est vu refuser l'agrément. Un autre a abandonné en cours de route, dérouté par la dimension invasive de l'enquête sociale. Deux d'entre eux ont vu leur agrément accepté, mais ne se sont pas engagés plus loin, inquiets du peu de chance que leur quête aboutisse tant les pays qui accueillent favorablement les demandes masculines sont rares.

La plupart ont également examiné l'hypothèse d'une coparentalité avec une amie ou un couple de lesbiennes. Certains s'y sont même engagés, mais tous ont reculé pour un faisceau de raisons que résume bien Henri :

On a très vite réalisé que pour un enfant, être baladé dans un foyer, puis dans un autre, c'est créer une situation d'enfant de divorcés. Donc ça, ça nous a un peu inquiétés. Finalement, on a fait définitivement une croix sur la coparentalité. Parce qu'élever des enfants à deux parents, ce n'est déjà pas évident, mais élever des enfants à quatre parents, c'est pratiquement impossible. On ne sera jamais d'accord sur tout. Même si on se connaît très bien et qu'on s'entend à priori bien, une fois que l'enfant est là, on ne s'entendra pas. Il y a beaucoup de couples hétéros qui basculent quand les enfants arrivent, alors que tout allait bien avant, donc on s'est dit, là, c'est aller au-devant des problèmes. On a pensé, en plus, qu'en cas de conflit, très probablement, c'est le couple des femmes qui aurait la garde complète des enfants. Donc, on s'est dit, on ne va pas faire des enfants pour ne pas les voir, ne pas les élever.

Et puis, tous entendent des histoires de coparenté dans lesquelles les pères sont en partie ou totalement évincés par les femmes, au nom de la compétence maternelle, notamment dans les premiers mois, voire les premières années. Pour des aspirants à la paternité à temps plein, ce scénario parait franchement périlleux.

Dès lors, la gestation pour autrui leur semble la meilleure voie. Celle qui leur permet d'être totalement présents auprès de l'enfant dès la naissance, qui leur accorde la garde 
exclusive tout en satisfaisant aussi le désir de transmission de ceux pour qui cette dimension de l'enfantement importe.

Mais, comme le dit Henri, si la gestation pour autrui se présente clairement comme la meilleure configuration, elle n'en est pas moins problématique : «Oser faire un enfant sans mère », voilà le défi auquel sont confrontés ces nouveaux parents.

\section{Maternité (s)?}

\subsection{GPA ou MPA?}

Dans les années 1990, seule existait la «procréation pour autrui», dite aussi «maternité pour autrui » (MPA). La femme qui portait un enfant pour une autre femme, était inséminée artificiellement avec le sperme du père et était donc enceinte de son propre enfant biologique. Aujourd'hui, du fait des progrès réalisés en matière de fécondation in vitro, la MPA se rencontre exceptionnellement: l'enfant est généralement issu des gamètes des parents intentionnels et se trouve de ce fait génétiquement étranger à la gestatrice. On parle alors de gestation pour autrui (GPA). L'expérience des agences américaines avec les couples hétérosexuels les a progressivement conduites, pour protéger les femmes porteuses d'un investissement maternel vis-à-vis de l'enfant, à proposer systématiquement une GPA aux couples d'hommes. Et donc de recourir à une gestatrice et à une donneuse d'ovocyte.

La GPA s'impose aux couples hétérosexuels comme une solution plus satisfaisante que la MPA, puisqu'elle leur permet d'engendrer un enfant issu de leurs deux corps. En revanche, opter pour l'une ou l'autre ne va pas de soi pour les couples d'hommes. Ceux qui veulent faire appel à une seule et même femme pour porter leur enfant et le concevoir se heurtent aujourd'hui à la réticence des agences. Certains ont accepté ce qui leur était proposé, malgré leur souhait premier, d'autres n'y avaient pas vraiment réfléchi, d'autres enfin ont insisté pour ne pas recourir à plus d'une personne. Dissocier ou ne pas dissocier la maternité biologique de la gestation? Les motivations ou les justifications a posteriori de l'option finalement retenue éclairent les représentations que ces hommes se font de la maternité.

\subsubsection{Ne pas dissocier : le choix de la simplicité pour l'enfant}

Quatre couples avec enfant, un père célibataire et un couple en projet ont choisi de ne recourir qu'à une seule femme. Ils motivent leur décision par le souhait de réduire la complexité de l'histoire qui a présidé à la naissance de l'enfant. Ils sont troublés par l'idée de deux mères, se demandent laquelle des deux est la « vraie » mère ou estiment qu'il sera trop compliqué d'expliquer à l'enfant le rôle de la donneuse. Pour ces pères, même en l'absence d'une maman au quotidien, un enfant a toujours une et une seule mère, celle qui l'a mis au monde.

Frédéric, célibataire, deux enfants, justifie ainsi son choix :

Pourquoi la MPA? Parce que je me suis dit que pour le gamin ou la gamine à venir, ça sera déjà assez compliqué à expliquer, le contexte homosexuel, homoparental. Je ne vais pas rajouter une difficulté en plus à cet enfant et lui dire : tu n'as pas une mère, tu en as deux. J'ai estimé, à tort ou à raison, que c'était trop le charger.

Jean-Louis et Pierre, deux enfants de six ans et deux ans, ont commencé leur démarche six ans avant la naissance de l'ainé. Jean-Louis explique pourquoi la «maternité pour autrui » leur semblait préférable : 
À l'époque, je n'avais pas l'impression qu'il y avait une autre possibilité. Depuis, il y a eu d'autres pères qui ont démarré leur projet et qui ont commencé à avoir une donneuse d'ovocytes et une mère porteuse. Et là, nous nous sommes dit que nous ne serions peut-être pas capables de faire comme eux. [...] on s'est demandé mutuellement ce qu'on en pensait et je me rappelle avoir dit que j'aurais été vraiment très embarrassé pour dire laquelle est la mère. Avoir deux mères, ça me gênerait. Je ne suis pas très à l'aise avec l'idée d'une mère à deux têtes. S'il fallait choisir, laquelle des deux je choisirais? Si l'enfant me demandait qui est sa mère? J'aurais du mal à lui répondre laquelle. À l'époque, on s'était dit que si on ne savait pas répondre à cette question, il fallait absolument continuer à n'avoir qu'une seule mère.

Pour Didier, en couple avec Jaime, la donneuse d'ovocyte représente une maternité plus «vraie». Elle viendrait déqualifier la gestatrice qui ne serait plus tout à fait une mère. Ainsi, déclare-t-il : «Je ne me voyais pas trop expliquer à ma fille : tu as une maman, mais ta maman ce n'est pas vraiment ta maman, les œufs viennent... »

S'affranchir de la présence d'une mère au quotidien ne signifie pas s'affranchir de notre système exclusif de représentation de la maternité. Ce souci de la part de certains hommes de simplifier pour l'enfant l'histoire de sa venue au monde traduit une conception de la parenté dans laquelle chacun n'a qu'une mère, une seule.

\subsubsection{Dissocier pour qu'aucune ne soit une mère}

Un père célibataire, dix couples avec enfants et cinq couples en projet ont eu ou auront recours à une donneuse d'ovocyte. D'autres préoccupations émergent de leur discours. Qu'ils aient accepté ce que l'agence leur proposait ou qu'ils aient délibérément choisi la GPA, deux inquiétudes paraissent déterminantes pour éliminer la "maternité pour autrui » et leur faire préférer la contribution de deux femmes. La première inquiétude suscitée par une «maternité pour autrui » réside dans la crainte que la séparation d'avec l'enfant soit vécue comme un abandon. Celui-ci impliquant non seulement une souffrance ou un sentiment de culpabilité chez la gestatrice, mais aussi un risque de traumatisme chez l'enfant. Le fait que la gestatrice ne soit pas tout à fait la mère, puisque l'enfant n'est pas biologiquement le sien, permet d'éloigner le spectre de l'abandon. Que l'enfant puisse idéaliser davantage la figure maternelle, absente dans sa vie quotidienne, si gestation et lien biologique sont réunis dans la même personne constitue la deuxième inquiétude. En divisant la maternité en deux, aucune des deux femmes ne serait «complètement» mère, aucune n'aurait abandonné son enfant, aucune ne pourrait se prêter à l'idéalisation d'une mère absente au quotidien.

Jean, deux enfants nés de GPA, considère que la GPA est un don et non un abandon, mais redoute que l'enfant ne le perçoive pas de la même manière. Il explique : «Les femmes sont plus réticentes à abandonner un enfant qui leur est propre... Pour l'enfant, c'est plus simple de n'en avoir qu'une... mais il peut très bien dire d'une femme qui a un enfant et qui ne s'en occupe plus, qu'elle l'a tout simplement abandonné. »

Michel et Max sont ensemble depuis 20 ans, ont des jumeaux de 2 ans issus de deux embryons parmi plusieurs conçus par l'un ou par l'autre. Les deux hommes ne souhaitent pas savoir à qui les jumeaux sont reliés biologiquement. Ils estiment être deux pères indépendamment du statut biologique de leur paternité et pensent que les enfants seront ainsi plus facilement intégrés dans leur famille respective. Michel explique pourquoi leur choix de dissocier gestation et lien biologique s'imposait : 
Il pourrait y avoir plus de problèmes de détachement entre la mère, celle qu'il faut bien appeler la mère, puisque dans le cadre d'une MPA elle est à la fois mère biologique et elle a porté... Et en y réfléchissant, on s'est dit que c'était peut-être pas mal que celle qui donne son patrimoine génétique n'ait pas de sentiment de maternité, donc que psychologiquement elle le surmonte bien parce qu'elle n'est pas enceinte, qu'elle ne le porte pas. Et que celle qui porte, psychologiquement, n'a aucune difficulté, ou moins de difficultés, à le laisser aller parce qu'elle sait que ce n'est pas son enfant biologique.

Ignorer ou tenir pour négligeable le lien génétique paternel tout en exprimant de l'empathie pour la gestatrice qui souffrirait de se séparer de son enfant biologique signe peut-être un rapport ambigu au lien biologique. Un lien biologique dit sans importance, mais non sans incidence. D'une part, en avoir connaissance pourrait déséquilibrer l'égalité de paternité souhaitée dans le couple. D'autre part, transmettre son patrimoine génétique confèrerait davantage un statut de mère que d'avoir été enceinte.

Henri, père de jumelles de 4 ans, s'interroge :

Dans notre idée, des deux femmes qui nous ont aidés, la maman serait plus la donneuse d'ovocyte. Parce qu'on s'est dit que dans leur esprit, la maman sera peut-être la femme chez qui elles verront des traits de ressemblance. Elles voudront chercher si elles ont ses cheveux, son nez, ses yeux... donc, on s'est dit que si on devait donner le nom de maman à l'une des deux femmes, ce serait plus à la donneuse d'ovocytes.

La crainte que la gestatrice "traditionnelle » ne soit idéalisée en révèle une autre : l'enfant pourrait voir en elle, une "vraie » mère qui pourrait concurrencer les parents intentionnels. On trouve ici une représentation accordant une certaine importance au lien biologique et la crainte que les seuls "vrais » parents demeureraient le père biologique et la mère à la fois gestatrice et biologique, réduisant à néant le projet homoparental dans lequel les parents sont deux hommes.

Claude et Hugues, encore en projet, préfèreraient séparer la mère porteuse de la mère donneuse pour «minimiser les liens qui pourraient se créer si c'était la même personne. En dissociant mère porteuse et donneuse, précisent-ils, on se donne une chance supplémentaire qu'il y ait moins de problèmes d'attachement ».

Un autre enquêté, Clément, également en projet : «Au début, on pensait à une MPA, mais le fait qu'il y ait un dédoublement, ça permet d'alléger le poids que ça représente pour elle. Et pour l'enfant, il n'y a pas une mère quelque part. Ce sont deux femmes. Ça diminue la place fantasmée qu'elle pourrait avoir...»

Il est présumé que la gestatrice pourrait souffrir de la séparation si elle portait un enfant lié biologiquement à elle. Cette conviction va de pair avec l'idée que l'enfant pourrait ressentir l'histoire de sa naissance comme un abandon plutôt que comme un don. Dans ce contexte, le recours à une donneuse d'ovocyte permet de circonscrire la menace d'une mère " complète » prenant trop de place dans l'histoire de l'enfant. Cette option permet de conserver des liens privilégiés avec celle des deux «tiers de naissance » qui serait la moins « menaçante », la moins « vraie », la gestatrice.

\subsection{Des pères sans mère? Avec une mère? Avec deux mères?}

Priver un enfant de mère entraine davantage de réprobation sociale que priver un enfant de père. Être père sans mère, c'est transgresser une norme qui réserve aux 
femmes les soins au tout petit. Pourtant, la question de fonctions spécifiques attachées strictement à l'un ou l'autre sexe apparaît aujourd'hui discutable. L'autorité n'est pas davantage l'affaire des hommes que la tendresse et les soins, celle des femmes. Les pères gays, comme d'autres «nouveaux pères » privilégient le relationnel et l'affectif, sont compétents dans le domaine des soins aux enfants, même tout petits. Être père sans mère ne se ramène pas seulement à l'absence de mère au quotidien. C'est s'affranchir d'une autre norme, plus liée à notre système de parenté, qui interdit de devenir père autrement qu'en désignant la femme qui a porté l'enfant et qui est censée l'accréditer en tant que père.

Les discours des pères gays rencontrés sont traversés par les représentations actuelles de la famille et de la paternité. Obligés d'innover, ils hésitent entre plusieurs représentations. Ils passent d'une représentation de la parenté basée sur l'engendrement dans laquelle un enfant ne peut avoir qu'un père et une mère à une autre plus centrée sur l'éducation, les soins aux enfants, c'est-à-dire la parentalité. Une telle représentation permet d'envisager deux pères sans mère. Ces représentations ne s'excluent pas mutuellement et certains enquêtés les combinent. Ils peuvent par exemple expliquer qu'ils sont deux pères, parce qu'ils sont les parents au quotidien, et qu'il y a une mère, voire deux mères, parce qu'elles ont contribué à la venue au monde de leur enfant.

L'attribution d'un statut de mère aux femmes qui ont aidé ces hommes à devenir père dépend de trois paramètres. D'abord, le nombre de femmes impliquées joue sur la manière de les désigner. Une même femme qui a donné son ovule et a porté l'enfant est souvent désignée par un terme maternel : c'est la mère de leur enfant. Ensuite, la signification attribuée au lien biologique contribue à la désignation de la donneuse. Elle pourra chez certains être la « vraie » mère ou la mère biologique ou la mère donneuse, tandis que chez d'autres elle sera la donneuse sans connotation maternelle. Enfin, l'importance accordée à l'enfantement ou à la parentalité pour définir ce qui fait un parent influe non seulement sur la manière de désigner la gestatrice et la donneuse une mère, voire deux -, mais également sur les termes utilisés pour désigner le couple d'hommes. Sont-ils deux pères? Deux parents, mais un seul père? Ou bien un père avec son compagnon?

\subsubsection{Pas de maman}

Quatre couples en projet et quatre couples de parents estiment que leurs enfants n'ont (ou n'auront) pas de maman. Ceux qui sont déjà pères ont eu recours à deux femmes, une gestatrice et une donneuse. Ils ne nient pas l'existence de ces «tiers de naissance», mais considèrent qu'une mère est avant tout quelqu'un qui s'implique au quotidien dans les soins et l'éducation.

Thierry, 35 ans, en couple avec Gilles, 37 ans, décrivent leur projet : «Il n’y a pas de mère, pas de maman. Parce que sinon ce seraient ses enfants et pas les nôtres et elle voudrait les élever. Je pense qu'il y aura des mots à mettre là-dessus. J'avais imaginé qu'on pouvait faire une espèce d'album photos, photos et écritures mélangées. Il y aurait des photos de la donneuse, des photos de nous, des photos de la gestatrice... »

Clément, 30 ans, lui aussi en projet, ne considère aucune des deux femmes comme une mère :

Pour moi, non, elle n'est pas mère, ni elle ni la donneuse d'ovocytes. Parce que si elle est mère, c'est que quelque part, elle a abandonné son enfant, et pour nous, personne n'a abandonné son enfant. Elle n'est pas mère, donc elle n'a pas abandonné son enfant. Elle a aidé ces 
deux papas à réaliser leur rêve. On peut l'appeler par son prénom ou ce qu'on veut. Mais l'appeler une mère, c'est lourd.

Que ces femmes ne soient pas des mères n'empêche pas que des relations s'instaurent entre elles ou l'une d'elles, plus généralement, la gestatrice, et le couple d'hommes. Ces relations ne sont pas toujours limitées au temps de la gestation, elles peuvent perdurer et instaurent parfois un lien quasi familial.

Max et Michel ont des jumeaux de deux ans. À leurs yeux, ce qui compte, c'est le projet parental, leurs enfants ont donc deux pères et pas de mère. Max explique :

Nous ne privons pas l'enfant d'une mère. Une mère classique donne son patrimoine génétique, accouche et élève. Nos enfants ne se construiront pas avec le fantasme d'une mère idéale, puisqu'on leur expliquera... Ils sont dans une situation où des pères les ont désirés, les ont aimés... Pour être très franc, aucune n'est réellement mère, c'est très clair. Mais il y a des femmes qui ont participé au miracle de la vie.

Quand ils parlent de la gestatrice, ils l'appellent par son prénom ou disent la donneuse, la porteuse, la surrogate. Max explique qu'il utilise sciemment ces termes parce qu'il ne veut pas employer le mot mère pour aucune des deux. Mais paradoxalement, il ajoute : «C'est plus qu'une amie pour nous, c'est une sœur. » De fait, ils sont restés plusieurs mois aux États-Unis après l'accouchement. Le contact avec elle est permanent par internet et ils se voient au moins une fois par an, soit c'est elle qui vient en France, soit c'est eux qui voyagent aux États-Unis.

\subsubsection{Une mère ou presque}

Un couple en projet, six couples de parents et un père célibataire accordent une certaine importance au modèle exclusif de la parenté. Damien et Christophe ont un petit garçon de trois mois au moment de l'entretien. Même si le lien biologique n'a pas été crucial pour décider qui serait le père, même s'ils partagent à parts égales les biberons et les couches, à leurs yeux « ça ne se peut pas d'avoir deux papas ».

Ils expliquent que la gestatrice " est la mère, puisqu'elle lui a donné naissance, mais qu'en même temps ce n'est pas la mère parce qu'elle ne va pas l'élever. Elle lui a donné naissance, elle l'a porté et elle sera toujours là... » Ils sont très proches de la gestatrice, de son mari et de leurs enfants. Il y a des photos partout dans leur appartement. Les filles de la gestatrice appellent l'enfant leur surrogacy brother ${ }^{5}$. Un lien quasi familial s'est noué tant du côté de la gestatrice que du leur. Pendant la grossesse, ils sont allés la voir outre-Atlantique pratiquement tous les mois. Pour l'accouchement, ils sont restés cinq semaines. Ils échangent très souvent sur internet, et souhaitent que cette relation s'installe dans la durée. Ils projettent d'avoir un deuxième enfant avec elle.

Pour certains, le père et la mère sont nécessairement ceux qui ont contribué à donner la vie. Jaime et Didier ont fait leurs les représentations psychanalytiques. Ils se montrent convaincus de la nécessité d'un père et d'une mère pas tant au sens de l'exercice de fonctions et de responsabilités parentales qu'au sens de la "scène primitive » et de "l'ordre symbolique ». Ils estiment que leur fille de deux ans a un papa, un seul, Didier, et une maman, une seule, même si elle a aussi un autre parent, Jaime, qui n'est ni un père ni un papa :

5 Frère de GPA. 
Elle a un père et une mère, et moi, je ne suis pas un beau-père, je ne suis pas papou ou pépé. Je suis Jaime. Je l'ai eu en même temps que Didier et je serai toujours là... Un enfant, pour se construire, doit avoir intériorisé le concept d'avoir un père et une mère. Ce concept, il faut l'avoir pour se construire soi-même. Dire à un enfant qu'il n'a pas de mère, qu'il n'a que deux pères, c'est vraiment selon nous, ce qu'il ne faut pas faire... De toute façon, il lui faudra une maman, parce qu'il faut bien qu'elle se représente comment elle a été conçue. L'idée, c'est la conceptualisation de la scène primitive. Il faut qu'elle puisse le concevoir, même si c'était médical. C'est pour ça que je pense qu'il faut lui dire qu'elle a une maman, mais qu'elle est très loin et qu'elle n'a pas pu l'élever...

Ils ont souhaité une «maternité pour autrui » justement pour pouvoir lui parler d'une maman. Didier explique qu'ils ont choisi une femme qui lui plaisait physiquement pour pouvoir le dire à l'enfant. Ils précisent qu'ils maintiennent des liens avec elle non pas tant parce qu'ils ont noué une réelle amitié que dans le but de pouvoir répondre aux demandes éventuelles de leur fille. Ils sont inquiets, craignent qu'elle ne vive son histoire comme un abandon et veulent consulter un pédopsychiatre et un psychanalyste pour mieux aborder cette question.

Les représentations de Marc se rapprochent de l'enfantement et de la parenté «naturels». La question de l'abandon ne se pose pas vraiment pour lui et son compagnon, car la gestatrice reste présente dans leur famille en tant que mère. Même loin, c'est une maman. Ils lui ont demandé l'autorisation que leur fille l'appelle maman. Elle a acquiescé après consultation de son mari et de ses enfants. Marc parle en anglais à sa fille pour symboliser le lien avec la mère américaine. Il explique :

Je veux lui offrir le plus de normalité possible. Je me dis que demain elle va être montrée du doigt à l'école. Si un jour elle se sent en difficulté, elle aura la possibilité de parler de son père et de sa mère. Si elle n'a pas envie de parler de moi, elle est libre de ne pas en parler. Si elle veut un accès à ses origines, elle l'aura toujours. Je voudrais lui offrir la structure familiale la plus normale possible dans un contexte anormal.

Mais au-delà des représentations, et de l'intention, c'est pour Marc et son compagnon le vécu d'une relation intime et intense avec la gestatrice qui la positionne en mère. «Mère porteuse, c'est un terme que je déteste, parce qu'elle est tellement plus que ça. Elle n'est pas une mère porteuse, c'est une amie, c'est la mère de notre enfant, elle a fait le don d'elle-même, d'un bébé qu'elle a porté, son propre enfant, avec qui elle a une ressemblance forte. [...] »

\subsubsection{Deux mères ou plus}

Un couple en projet et trois couples de parents s'éloignent d'un modèle exclusif de la parenté et évoquent plus d'une mère.

Edgar et Richard sont les deux pères de jumeaux. Edgar est le père biologique et légal et Richard est leur père adoptif selon la loi californienne. Les jumeaux, une fille et un garçon, ont une mère porteuse, mais sont issus de deux donneuses différentes. La fille a une donneuse connue et amie; le garçon, une donneuse américaine théoriquement anonyme avec laquelle les pères essaient de garder un lien. Pour eux, il ne fait pas de doute que ces trois femmes sont des mères, l'une porteuse et les deux autres biologiques. Ce qui préoccupe davantage les deux pères réside dans le déséquilibre entre les deux enfants, l'une pouvant connaître sa mère biologique, l'autre non. 
Edgar explique : «Le rôle de la mère traditionnelle est réparti entre plusieurs personnes. La donneuse de notre fille accepte de jouer le rôle de mère biologique pour elle et de mère sociale pour les deux enfants. »

Richard et Edgar ne se sentent pas menacés dans leur paternité par l'existence de ces femmes présentes pour l'une dans leur proximité, pour les autres, par webcam ou dans les albums de photos. La représentation à l'œuvre ici est que toute femme, donneuse ou gestatrice, qui a contribué à donner la vie est une mère.

Les motivations ou les justifications a posteriori du recours à une seule personne ou bien à deux personnes révèlent les représentations sociales attachées à la gestation et au lien biologique. Dans les deux cas, les mêmes conceptions de la maternité dans lesquelles les dimensions gestationnelle et biologique font une mère " complète » sont paradoxalement à l'œuvre. Dans un cas, ces représentations sont rassurantes, dans l'autre cas, elles sont menaçantes.

Que ces femmes soient désignées comme des mères, des pas tout à fait mères, ou pas du tout des mères, elles font partie de l'histoire de l'enfant. Elles sont des «tiers de naissance » avec lesquels certains hommes construisent une relation qu'ils envisagent ou non de maintenir au-delà de la grossesse. Quelques-uns mettent l'accent sur le fait que la mère porteuse et son mari partagent les mêmes valeurs et les mêmes options politiques. D'autres insistent sur l'amitié qui s'est développée pendant le processus. D’autres encore décrivent des liens quasi familiaux.

\section{Conclusion}

\section{L'expérimentation de nouveaux liens familiaux}

Pères en solo, deux pères à égalité de rôle, un père et son compagnon qui le seconde : les couples gays ayant recours à la GPA ont à élaborer, choisir, dénommer la place de chaque partenaire dans l'engendrement, puis dans l'engagement éducatif auprès d'enfants accueillis dans ce cadre hors norme. Écloses en une période d'infléchissement de la paternité vers davantage d'engagement quotidien, de valorisation de l'affectif et du relationnel, les paternités gays profitent de ces transformations contemporaines tout en contribuant à ébranler encore plus l'édifice. Invités à réfléchir sur leur désir d'enfant, les couples homosexuels masculins deviennent moteurs de projets de vie qui se différencient des modèles habituels tout en en étant tributaires.

Familles sans mère ou dotées d'une mère de naissance, voire de deux mères d'engendrement, la représentation habituelle de la maternité vacille sur son socle. Voilà désormais des foyers dont est absente, au quotidien mais aussi dès la conception de l'enfant, une personne féminine valorisée. En effet, dans ces nouvelles configurations, la femme (les femmes) ayant contribué à la venue de l'enfant n'a pas une place acquise. Prestataire d'un service, maintenue hors du halo familial ou personne reconnue en son sein? Figure lointaine et détachée ou figure maternelle avec laquelle se nouent des relations de complicité et d'affection? Personnage occupant une place dans le réel parental et enfantin par le canal de la circulation des informations, des photos, par le truchement des rendez-vous de vacances ou personnage abstrait ayant une place désignée au tout début de l'histoire, mais qui n'est pas invité à accompagner même de loin la suite de l'aventure? La place de la femme ou des femmes qui ont participé à l'enfantement se dessine et se définit sous de multiples facettes.

Notre enquête met à jour le caractère véritablement expérimental de ces nouvelles modalités d'entrer en paternité. Notre approche de ce monde jusqu'alors invisible 
s'inscrit dans le prolongement et la continuité de l'enquête et de la réflexion menées par Emmanuel Gratton auprès de parents gay adoptifs ou en coparentalité tout en découvrant peut-être une émancipation plus affirmée par rapport au moule familial traditionnel. Shiren Kashmeri, dans sa thèse sur la GPA en Ontario, évoque, comme Emmanuel Gratton, l'hypothèse que le recours à une mère porteuse devienne à l'avenir, en dépit de son coût et de sa complexité, une démarche de plus en plus fréquente pour les gays aspirant à fonder une famille.

Annonciatrices d'un déclin de la toute-puissance maternelle ou nouveaux scénarios de familles multicomposées? À l'aune de ces inventions et au fil de leurs incertitudes s'esquisse un panorama encore renouvelé de la famille contemporaine. Car ces innovations ne surgissent pas dans une société figée. Bien au contraire.

$\mathrm{Au}$ cours des dernières décennies, les configurations familiales se sont diversifiées à vive allure. La famille nucléaire dans laquelle parenté légale, éducative et biologique repose sur les mêmes épaules, celles du couple hétérosexuel procréateur, ne représente plus le modèle majoritaire même s'il demeure dominant. Les familles adoptives ou d'accueil ont forgé depuis longtemps des scénarios où la parenté de naissance ne concorde pas avec la parenté éducative. Plus récemment, les familles recomposées issues de la montée du démariage fleurissent, installant dans le paysage des regroupements domestiques où les beaux-parents, nouveau conjoint de la mère ou nouvelle compagne du père, sont présents au quotidien dans l'éducation d'enfants qui ne sont pas issus de leurs gènes. Les procréations médicalement assistées, dès qu'elles font appel à un tiers donneur (de sperme ou d'ovocyte), complètent ce tableau dans lequel un des deux parents du quotidien n'est pas le géniteur des enfants qui vivent avec lui. Les familles homoparentales ajoutent une nouvelle touche à cette mosaïque de combinaisons où procréation et responsabilité parentale ne coïncident pas.

Psychologues, sociologues, anthropologues se sont attentivement penchés sur le berceau de ces nouveaux foyers, proposant de troquer une conception univoque calquée sur le modèle de la famille nucléaire contre une représentation qui prend acte de cette démultiplication du lien familial. Ils ont accordé crédit à la capacité de la société à penser ses évolutions sociales. La famille homoparentale, avec les autres familles multicomposées, invite à considérer la dimension plurielle et mouvante de ces autodéfinitions. Les parents gays s'inscrivent dans ce mouvement qui tente de définir une place à ces constructions d'enfantement impliquant plus de deux acteurs. Place parentale stricte ou seulement symbolique, positionnement relationnel et affectif ou uniquement historique, les scénarios varient. Ces familles qui ne se limitent pas au face-à-face entre le couple parental et sa descendance densifient le halo de la famille élargie ou composée moderne accueillant des personnes attachées entre elles par des relations diverses, abstraites ou proches, réelles ou fantasmées. Tandis que paternité et maternité se redéfinissent en se redéployant, la famille se met à l'épreuve de la plasticité et de la mobilité des mœurs. L'homoparentalité, et notamment lorsqu'elle se conjugue au masculin, s'inscrit dans ce mouvement de remodelage et de subjectivisation du lien familial. 


\section{Bibliographie}

Aplg. 2007. Guide bibliographique de l'homoparentalité, Paris, APGL.

Berkowitz, D. et W. Marsiglio. 2007. « Gay Men: Negotiating Procreative, Father, and Family Identities », Journal of Marriage and Family, vol. 69, no 2, p. 366-381.

Borrillo, D. et P. Lascoumes. 2002. Amours égales. Le Pacs, les homosexuels et la gauche, Paris, La découverte.

Cadoret, A. 2000. "L'homoparentalité, construction d'une nouvelle figure familiale », Anthropologie et Sociétés, vol. 24, no 3, p. 39-52.

Cadoret, A. 2002. Des parents comme les autres, Paris, Odile Jacob.

Delaisi de Parseval, G. 1981. La part du père, Paris, Le Seuil.

Delaisi De Parseval, G. 2008. Famille à tout prix, Paris, Le Seuil.

Delumeau, J. et D. Roche. 2000. Histoire des pères et de la paternité, Paris, Larousse.

Desjeux, C. 2006. Homosexualité et procréation: les prémices d'un matriarcat?, Paris, L'Harmattan.

Dubreuil, E. 1998. Des parents de même sexe, Paris, Odile Jacob.

Eribon, D. 1999. Réflexions sur la question gay, Paris, Fayard.

Girard, C. 2006. «Père comme les autres», Paris, Hachette.

Gratton, E. 2008. L'homoparentalité au masculin. Le désir d'enfant contre l'ordre social, Paris, PUF.

Gross, M. 2006. «Désir d'enfant chez les gays et les lesbiennes », Terrain, no 46, p. 151164.

Gross, M. 2009. L'homoparentalité, Paris, Les idées reçues, Le Cavalier Bleu.

Héfez, S. 2007. Dans le coeur des hommes, Paris, Hachette Littérature.

Kashmeri, S. 2008. Unraveling Surrogacy in Ontario, Canada. An Ethnographic Inquiry on the Influence of Canada's Assisted Human Reproduction Act (2004), Surrogacy Contracts, Parentage Laws, and Gay Fatherhood, Master of Arts, Montréal, Concordia University.

Lev, A. 2006. « Gay dads: choosing surrogacy », Lesbian and Gay Psychology Review, vol. 7, no 1, p. 73-77.

Lewin, E. 2009. Gay Fatherhood, University of Chicago Press.

Mehl, D. 2008. Enfants du don, Paris, Robert Laffont.

Ragoné, H. 2000. «Of Likeness and Difference: How Race is Being Transfigured by Gestational Surrogacy», dans Ideologies and Technologies of Motherhood: Race, Class, Sexuality, Nationalism, sous la dir. de H.R.A.F.W. Twine, New York, Routledge, p. 56-75.

Stacey, J. 2006. "Gay Parenthood and the Decline of Paternity as We Knew It », Sexualities, vol. 9, no 1, 02, p. 27-55.

Tarnowski, F. 2010. Etre père et homosexuel dans la France contemporaine, thèse de doctorat en anthropologie sociale, Toulouse, EHESS.

Teman, E. 2010. Birthing a mother. The surrogate body and the pregnant self, University of California Press. 